\title{
Partial Discharge Detection and Characteristics of Gas-insulated Substation Free Metal Particles Based on Acoustic Emission
}

\author{
Runjie Shen, ${ }^{1}$ Yanghui Ou, ${ }^{1}$ Ying Zhou, ${ }^{2}$ Weishi Zhao, ${ }^{3}$ and Xuefeng $\mathrm{Li}^{1 *}$ \\ ${ }^{1}$ College of Electronic and Information Engineering, Tongji University, \\ 4800 Cao'an Highway, Shanghai 201804, P. R. China \\ ${ }^{2}$ Research Institute of Structural Engineering and Disaster Reduction, Tongji University, \\ 1239 Siping Road, Shanghai 200092, P. R. China \\ ${ }^{3}$ East Branch Natural Gas Marketing Company, PetroChina Company Limited, \\ No. 1200 Century Avenue Pilot Free Trade Zone, Shanghai 200135, P. R. China
}

(Received July 4, 2018; accepted March 13, 2019)

Keywords: gas-insulated substation, partial discharge, acoustic emission, free metal particles

Metal particles can easily be produced in the production and installation of a gas-insulated substation (GIS). The partial discharge (PD) induced by metal particles is a significant security risk to GIS. At present, some related research studies focus on the PD of individual metal particles, but less attention is paid to that of accumulated metal particles. In this study, the PD of the accumulated metal particles was investigated. A defect model of surface free metal particles on a basin-type insulator was set up. Four models of free metal particles of different sizes lying inside the GIS were designed. PD signal waveforms were measured using acoustic emission (AE) sensors. The frequency spectrum characteristics of the PD signal were analyzed, and the PD characteristics under different voltage waveforms were compared. Research results showed that, through the detection of PD signals, the accumulation of free metal particles on the surface of the insulator could be predicted and evaluated to ensure the safe operation of GIS equipment.

\section{Introduction}

With the wide application of gas-insulated substations (GISs), the operation reliability of GIS has aroused widespread concern in the international community. Over the past decades, it has generally been considered that GIS is a device that does not require maintenance or a very long maintenance cycle. However, according to 40 years operation experience, the insulation fault is always one of the most important factors affecting the reliability of GIS. ${ }^{(1,2)}$ The insulation fault is caused by defects such as surface free metal particles, high-voltage conductor spikes, insulator surface contamination and internal defects, shell spikes, and floating electrodes. In 1996, the International Council on Large Electric systems did a survey from customers worldwide. The results of the survey showed that the fault rates caused by different types of insulation defect were different, and that the insulation fault rate caused by the metal particles and foreign body was the highest. ${ }^{(3)}$ Partial discharge (PD), the early manifestation of insulation

*Corresponding author: e-mail: lixuefeng@tongji.edu.cn https://doi.org/10.18494/SAM.2019.2267 
fault within GIS equipment, carries important information about GIS defects. If PD is detected and removed as early as possible during operation, the equipment fault rate will be reduced and the power system reliability will be improved.

At present, a lot of research studies have been carried out for the PD phenomenon of metal particles in GIS. ${ }^{(4-6)}$ Prabakaran et al. used an actual $420 \mathrm{kV}$ GIS as an experimental platform to study the discharge characteristics of the particles fixed on a high-voltage bus bar, an outer casing, and an insulator. ${ }^{(7)}$ Holmberg et al. used the particle suspension time distribution to simulate the particles and considered the particle suspension time to evaluate the particle discharge characteristics. ${ }^{(8)}$ Runde et al. studied the relationship between the amplitudesuspension time characteristic spectrum of an ultrasonic signal and the particle length, and used the maximum measured amplitude of the ultrasonic signal as the characteristic parameter. ${ }^{(9)}$ In addition, Schlemper et al. studied the characteristic spectrum of the particle discharge amplitude-suspension time and analyzed the effect of charge-mass ratio on the particle suspension time. ${ }^{(10)}$ Current research studies mainly focus on the PD of a single particle. However, multiple metal particles may be produced during the production and installation; unfortunately, there have been no public reports about such issues yet. Therefore, whether the number of particles would affect their PD characteristics, the PD regularity of multiple particles should be examined.

In view of the above problems, four metal particle models were designed to imitate metal particle accumulation and the PD of these models was observed; the PD regularities of these models were also examined in this study. Research results show that the characteristics of a PD-induced acoustic emission (AE) signal and the size of metal particles have an obvious correlation, which can be used as the basis for determining the degree of insulation structural damage. It is also valuable for the in situ detection of high-voltage equipment such as transformers.

\section{Principle Analysis}

The classical PD theory is based on the theory of electronic avalanche or flow column. This theory can explain the microsecond single pulse phenomenon well. It has also achieved great success in the electrical pulse method. However, a large number of studies show that PD is a nanosecond discharge process, and its time domain waveform is composed of multiple continuous and amplitude-decimated pulses. Under this circumstance, the collisional ionization or flow column theory cannot explain the oscillation attenuation characteristics, and further research on the generation of $\mathrm{PD}$ is required.

Generally, the equivalent circuit of discharge can be represented by a second-order circuit that consists of a capacitor, an inductor, and a resistor. The capacitance and inductance are constants that do not change with time, and the resistance is nonlinear in terms of time; all three are lumped parameters. The characteristics of the discharge depend on the inherent characteristics of the resistance and discharge loop. The equivalent of the resistance to the linear resistance is a classical research method widely used to calculate the discharge pulse circuit with resistance. ${ }^{(12)}$ The discharge current of the equivalent circuit is shown as 


$$
i=i_{0+} e^{-\delta t} \cos w t
$$

Here, $i$ is the discharge current, $i_{0^{+}}$is the initial current, $\delta$ is a constant, $w$ is the oscillation frequency, and $t$ is the discharge time. Since the discharge moment is an electron avalanche or flow column theory, $i_{0+}$ is considered to be equal to $i_{\max }$; the above parameters are shown as

$$
\left\{\begin{array}{c}
i_{0+}=\frac{u_{0}}{\omega L}, \\
\delta=\frac{R}{2 L}, \\
\omega=\sqrt{\frac{1}{L C}-\left(\frac{R}{2 L}\right)^{2}} .
\end{array}\right.
$$

The parameters in the circuit are related to the discharge circuit. According to the results of the theoretical analysis above, when discharge occurs, a damped oscillation is generated in the equivalent discharge loop. Under the effect of the damped oscillation, the corresponding ultrasonic wave is generated in the medium. A large number of experiments show that the frequency of the PD signal is distributed in the $20-110 \mathrm{kHz}$ range. Therefore, we select sensors in the same frequency band for PD signal acquisition.

\section{Experiments}

The detection system is built as shown in Fig. 1. It consists of a voltage source to provide voltage for defect models, an $\mathrm{AE}$ sensor with a resonant frequency of $30 \mathrm{kHz}$, the $10 \mathrm{~dB}$ frequency bandwidth of which is $20-110 \mathrm{kHz}$ to collect PD signals, a preamplifier with a gain of $40 \mathrm{~dB}$ and a bandwidth of $10-50000 \mathrm{kHz}$ to amplify the signals collected from the AE sensor, and a PC for data acquisition and processing.

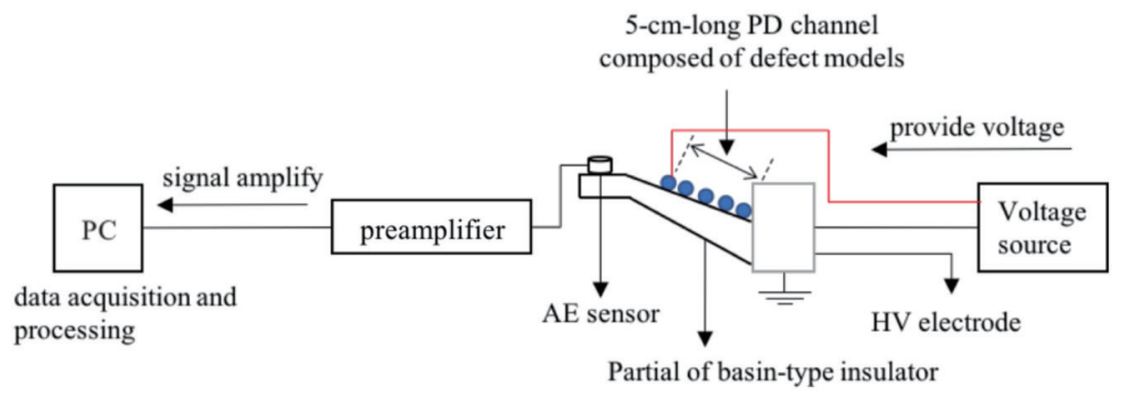

Fig. 1. (Color online) Detection system. 
Four defect models of the free metal particles, which use copper beads with diameters of 1.0 (model 1), 1.8 (model 2), 2.0 (model 3), and 2.5 (model 4) mm, are shown in Fig. 2(a). They are arranged in series. As shown in Fig. 2(b), a 5-cm-long PD channel is formed by an insulating double-sided adhesive on the surface of the basin insulator.

Four sets of experiments were carried out through the experimental platform. In each group of experiments, three PD initial voltage measurements were performed and averages were obtained to determine the initial PD voltages of the four different defect models. Experimental results show that the PD defect models 1-4 produce PD signals at initial voltages of 7.02, 6.13, 4.05, and $2.57 \mathrm{kV}$, respectively.

\section{PD Signal Analysis}

In this paper, time and frequency domain analyses are used to analyze the PD signals generated by the four defect models. AE signals are extracted by these two methods. We selected root mean square (RMS) as the feature parameter in the time domain. It can be calculated as

$$
R M S=\sqrt{\frac{\sum_{i=1}^{N} X_{i}^{2}}{N}} .
$$

Here, $N$ is the number of sampling points of the AE signal and $X_{i}$ is the amplitude corresponding to the sampling point of the $i$-th AE signal. The maximum power spectrum (MPS) is selected as the feature parameter in the frequency domain that can be calculated as

$$
M P S=\max [p s d(f)], f \in(1, N) .
$$

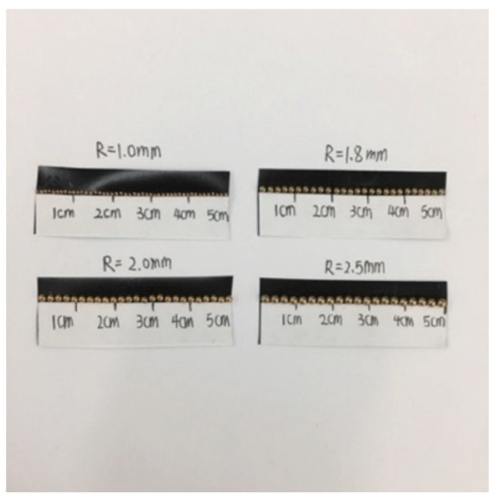

(a)

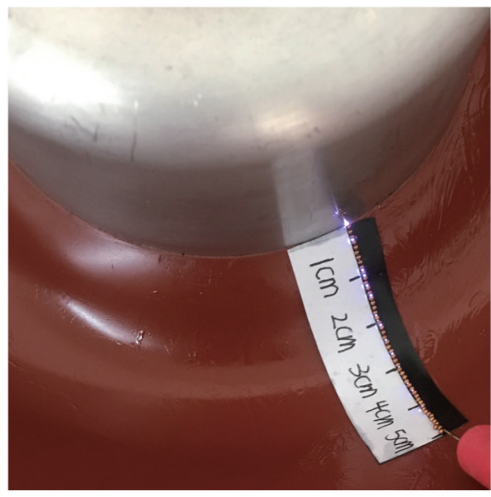

(b)

Fig. 2. (Color online) (a) Four defect models and (b) 5-cm-long PD channel. 


\subsection{Signal acquisition and denoising}

The PD signal is collected by the AE sensor, which is closely attached to the edge of the basin insulator. The sampling frequency is set to $1.0 \mathrm{MHz}$ through the data acquisition card, and PD signals are collected. Four groups of time domain signals and the frequency domain of 0-500 kHz obtained by fast Fourier transform (FFT) are shown in Figs. 3(a)-3(d).

By comparing frequency domain diagrams, the PD signals are found to be mainly concentrated in the $0-170 \mathrm{kHz}$ range. The acquired PD signals are processed by wavelet transform to obtain the time and frequency domain signals of the four sets of defect models as shown in Figs. 4(a)-4(d).

Through time domain signal analysis, the maximum amplitude of the PD signal generated by model 1 is found to be $0.55 \mathrm{~V}$, and that generated by model 2 is found to be $0.48 \mathrm{~V}$. This does not present many differences, and the amplitudes of the PD signals generated by these
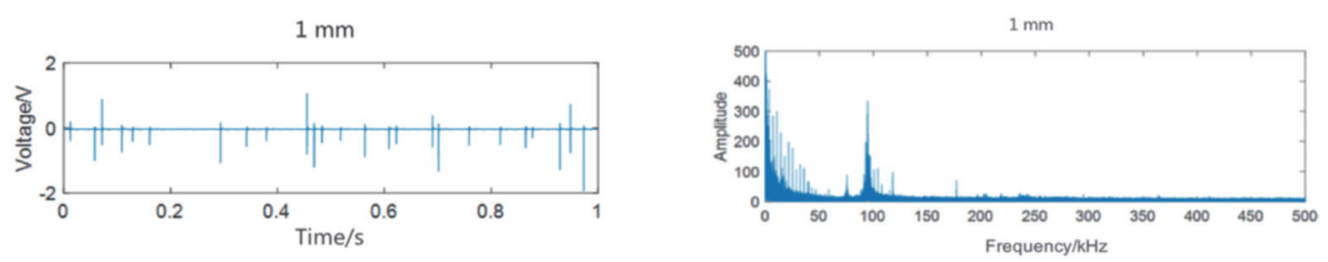

(a)
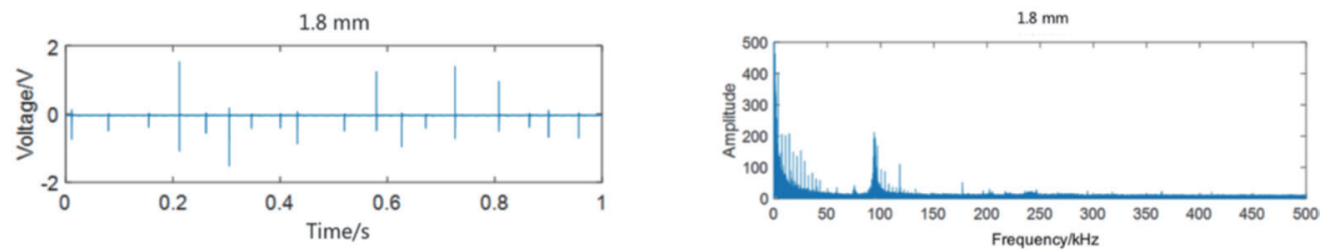

(b)
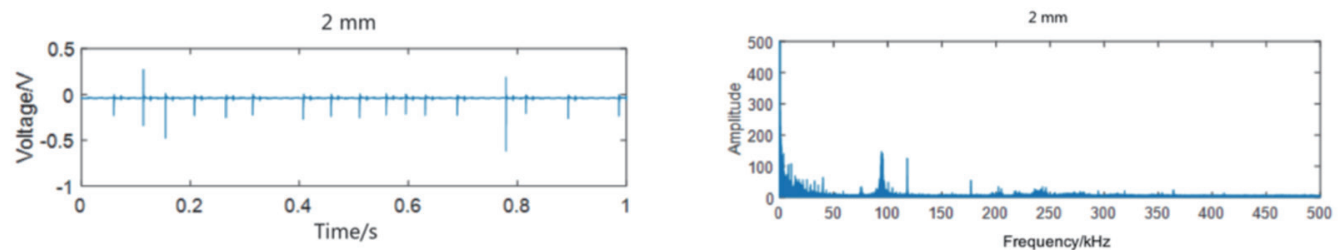

(c)
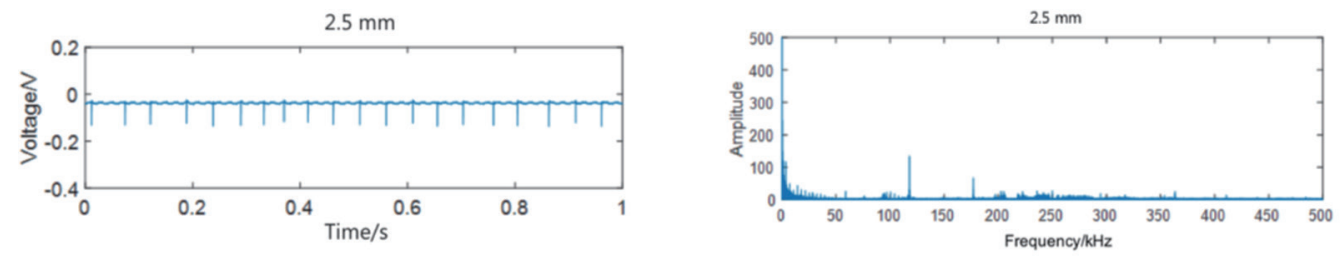

(d)

Fig. 3. (Color online) Time and frequency domain diagrams of models (a) 1, (b) 2, (c) 3, and (d) 4 at initial voltages of $7.02,6.13,4.05$, and $2.57 \mathrm{kV}$, respectively. 

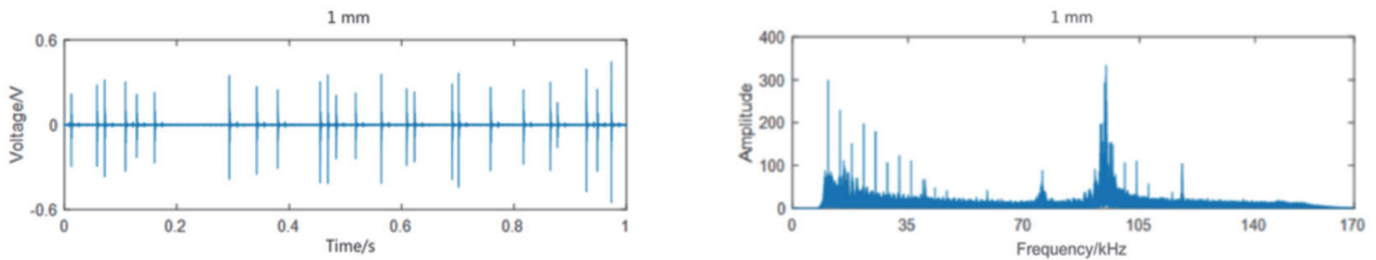

(a)
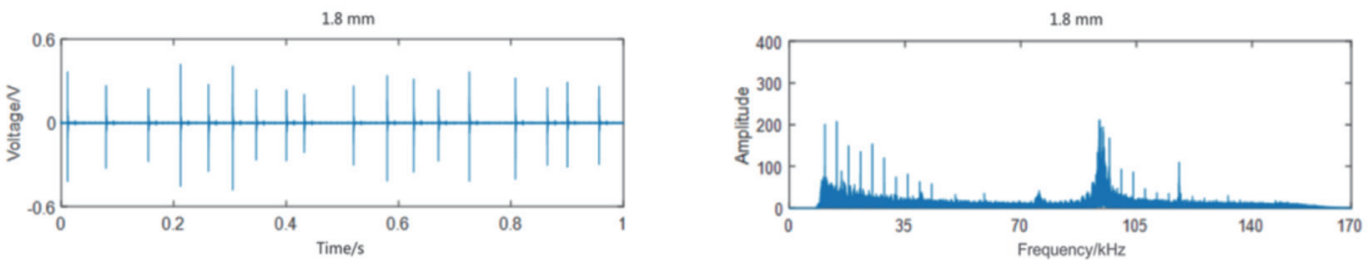

(b)
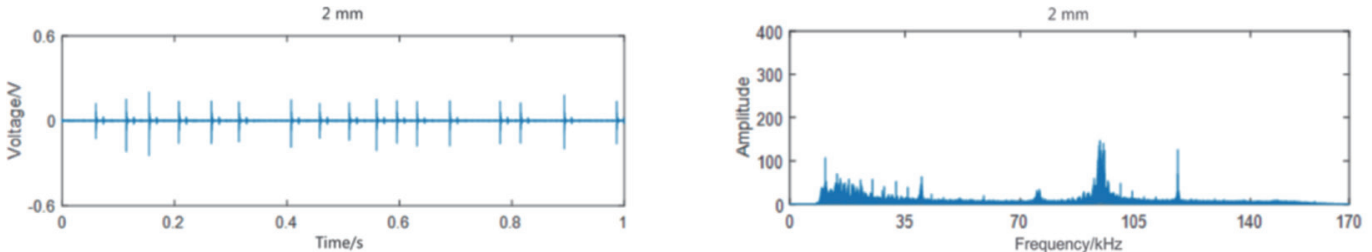

(c)
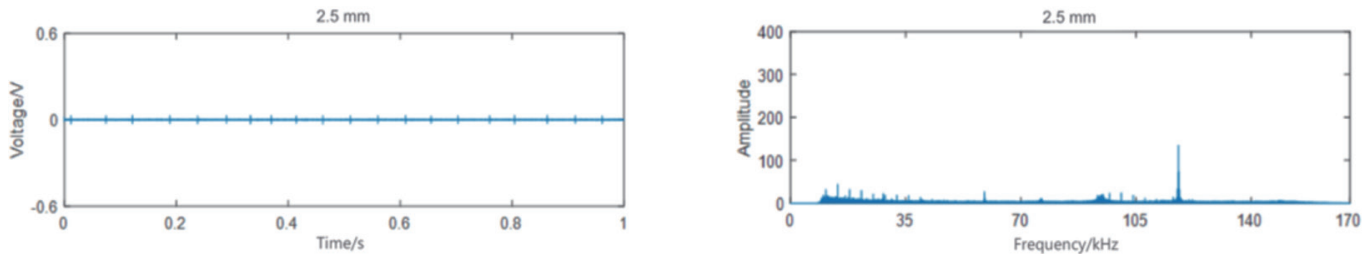

(d)

Fig. 4. (Color online) Time and frequency domain diagrams of models (a) 1, (b) 2, (c) 3, and (d) 4 for PD signals after wavelet denoising.

two models show obvious transitions with time. For models 3 and 4, the detected PD signal amplitudes are maximum at 0.24 and $0.03 \mathrm{~V}$, respectively. Compared with the PD signals generated by models 1 and 2, a large decrease in amplitude is observed for the signals generated by models 3 and 4 . Generally, the amplitude of the PD signal negligibly changes with time, especially the amplitude of the PD signal generated by model 4, which basically maintains a relatively stable range. The RMS values extracted for the four models are shown in Fig. 5.

By analyzing the RMS values, as the diameter of the copper bead in the defect model increases, the RMS is also found to increase, and the relationships between the RMS values and copper bead diameters of the four models are observed to be quadratic with a fitness of 0.9842 . To some extent, RMS can be an effective value of the PD signal. It can be considered that, when PD occurs, as the diameter of the copper bead in the defect model increases, the effective value of the PD signal increases. 


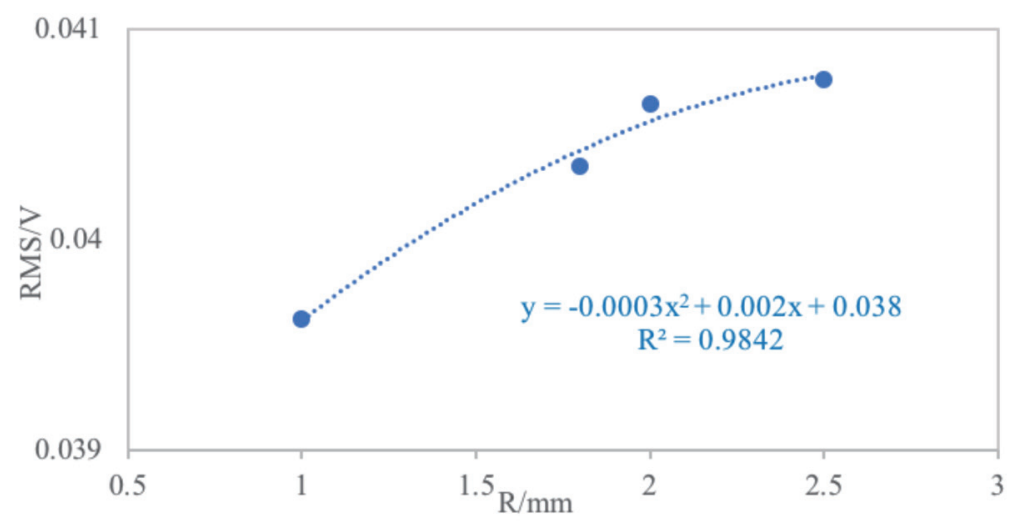

Fig. 5. (Color online) RMS values extracted for four defect models.

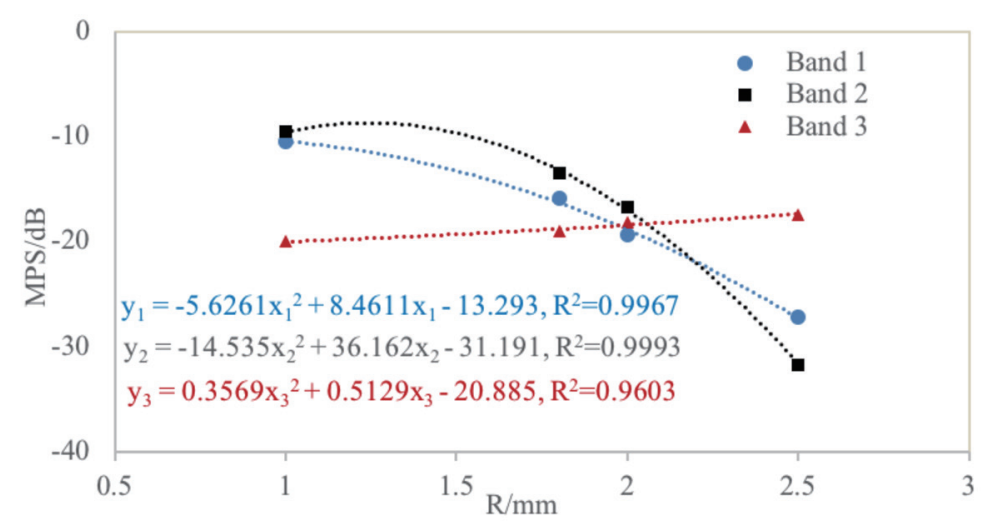

Fig. 6. (Color online) MPS values of three frequency bands for four defect models.

\subsection{Frequency domain analysis}

Through frequency domain signal analysis, it is found that the frequency spectra of the four defect models mainly include the following three frequency bands: frequency band 1 : 0-56 kHz, frequency band 2: $56-107 \mathrm{kHz}$, and frequency band 3: $107-170 \mathrm{kHz}$. It can be seen from the frequency domain diagram that the frequency spectrum distribution trend does not change with the diameter of the copper bead in the defect model, and that the energy that mainly concentrates in the frequency bands 1 and 2 decreases with the increase in the diameter of the copper bead in the defect model.

Figure 6 shows the MPS values of the AE signals for the four different defect models in the three frequency bands. In frequency bands 1 and 2, as the diameter of the copper bead in the defect model increases, the MPS value decreases; but in frequency band 3, as the diameter of the copper bead in the defect model increases, the MPS value increases. Among the models, in frequency bands 1, 2, and 3, the copper bead diameter has a binomial relationship with the MPS value, and the fitting degrees are $0.9967,0.9993$, and 0.9603 , respectively. This indicates that the four defect models have a PD signal of $0-170 \mathrm{kHz}$ with a maximum energy concentration of $0-107 \mathrm{kHz}$. 


\section{Conclusions}

In this study, we designed four defect models and a detection system to study the PD characteristics of these defect models. Observation results show that when PD occurs, the initial voltage of PD increases with the copper bead diameter, and the effective value of the PD signal also increases. For the four defect models, the PD signal was detected from 0 to $170 \mathrm{kHz}$, and the spectral distribution trend did not change with the copper bead diameter. The copper bead diameter and MPS value had the best quadrupling effect at $56-107 \mathrm{kHz}$. It can be inferred that, as the metal particles accumulate, the initial voltage required for local power generation decreases. At the beginning of PD, the weaker the detected PD signal, the more the accumulation of metal particles. In a future study, the eigenvalues of the four discharge signal models will be extracted and the PD signal will be identified by the SVM algorithm. We will analyze the accuracy of recognition before and after denoising and improve the recognition algorithm to achieve higher recognition accuracies.

\section{Acknowledgments}

This work is partially supported by the Fundamental Research Funds for the Central Universities (22120180121, 22120170260, and 22120180299) and the National Natural Science Foundation of China (Grant No. 61873189).

\section{References}

1 J. S. Pearson, O. Farish, B. F. Hampton, M. D. Judd, and D. Templeton: IEEE Trans. Dielectr. Electr. Insul. 2 (1995) 893.

2 X. Li, C. R. Li, and L. J. Ding: High Voltage Eng. 29 (2003) 26.

3 X. X. Zhang, N. Shu, X. G. Xu, X. Li, and J. Tang: Trans. China Electr. Soc. 30 (2015) 250.

4 B. Qi, C. Li, Z. Xing, and Z. Wei: IEEE Trans. Dielectr. Electr. Insul. 21 (2014) 766.

5 X. H. Wang, T. H. Li, D. Ding, and M. Z. Rong: IEEE Trans. Plasma Sci. 42 (2014) 2536.

6 H. X. Ji, C. R. Li, Z. K. Pang, G. M. Ma, X. W. Cui, W. J. Zhao, and J. Wang: IEEE Trans. Dielectr. Electr. Insul. 24 (2017) 259

7 T. Prabakaran, S. Usa, and A. S. Kumar: 2013 IEEE 1st Int. Conf. Condition Assessment Techniques in Electrical Systems (IEEE, 2013) 249.

8 M. E. Holmberg, M. L.-A. Sjoberg, and A. E. Vlasths: 9th Int. Symp. HV Engineering (1995) 5661.

9 M. Runde, T. Aurud, K. Ljokelsoy, L. Lundgaard, J. E. Nokleby, and B. Skyberg: IEEE Trans. Power Delivery 12 (1997) 714.

10 H. D. Schlemper and K. Feser: 10th Int. Symp. HV Engineering (1997) 87.

11 X. Li, Y. Shao, Y. Yu, Y. Zhang, and R. Shen: Sens. Mater. 29 (2017) 1305.

12 M. Cacciari, A. Contin, G. Rabach, and G. C. Montanari: IEE Proc.: Sci. Meas. Technol. 142 (1995) 102.

13 A. J. Reid, M. D. Judd, R. A. Fouracre, B. G. Stewart, and D. M. Hepburn: IEEE Trans. Dielectr. Electr. Insul. 18 (2011) 444.

14 L. Zhang, X. Han, and J. Li: IEEE Trans. Dielectr. Electr. Insul. 24 (2017) 296.

15 H. X. Ji, C. R. Li, Z. K. Pang, G. M. Ma, X. W. Cui, Z. P. Zeng, and Z. H. Rong: IEEE Trans. Dielectr. Electr. Insul. 23 (2016) 3355.

16 W. S. Gao, D. W. Ding, W. D. Liu, and X. H. Huang: IEEE Trans. Dielectr. Electr. Insul. 20 (2013) 782.

17 S. Okabe, G. Ueta, and T. Utsumi: IEEE Trans. Dielectr. Electr. Insul. 22 (2015) 2889.

18 H. F. Guo, F. S. Lu, and K. F. Ren: IEEE Trans. Dielectr. Electr. Insul. 21 (2014) 1942.

19 Z. C. Wu, Q. G. Zhang, J. Ma, X. A. Li, and T. Wen: IEEE Trans. Dielectr. Electr. Insul. 25 (2018) 1454.

20 X. J. Shao, W. L. He, J. L. Xu, M. X. Zhu, and G. J. Zhang: IET Sci. Meas. Technol. 12 (2018) 90.

21 S. Rudd, S. D. McArthur, and M. D. Judd: IEEE Trans. Dielectr. Electr. Insul. 17 (2010) 149. 
M. Hikita, S. Ohtsuka, J. Wada, S. Okabe, T. Hoshino, and S. Maruyama: IEEE Trans. Dielectr. Electr. Insul. 19 (2012) 8.

23 T. Ito, M. Kamei, G. Ueta, and S. Okabe: IEEE Trans. Dielectr. Electr. Insul. 18 (2011) 1847.

24 J. Tang, D. Yang, F. Zeng, B. W. Tang, K. Li, Q. Yao, and Y. L. Miao: IET Gener. Transm. Distrib. 12 (2018) 1240.

25 M. Ren, M. Dong, R. X. Ye, and Y. Liu: IEEE Trans. Dielectr. Electr. Insul. 23 (2016) 3593.

26 H. F. Guo, H. Y. Qiu, L. Yao, F. Huang, and K. F. Ren: IEEE Trans. Dielectr. Electr. Insul. 23 (2016) 1475

\section{About the Authors}

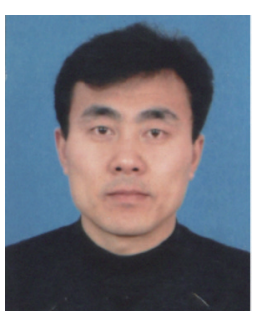

Runjie Shen received his B.S. degree from Xi'an Polytechnic University, China, in 1995. He received his M.S. degree from Xi'an Jiaotong University, China, in 1998 and his Ph.D. degree from Zhejiang University, China, in 2004. From 2004 to 2006, he was a postdoctoral researcher at Zhejiang University. In 2006, he became an associate researcher at Zhejiang University. Since 2011, he has been an associate researcher at Tongji University. His research interests are in sensors and NDT.

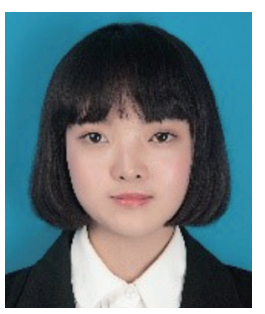

Yanghui Ou received her B.S. degree from Nanjing University of Posts and Telecommunications, China, in 2016. Since 2017, she has been studying at Tongji University for her M.S. degree. Her research interests are in sensors and NDT.

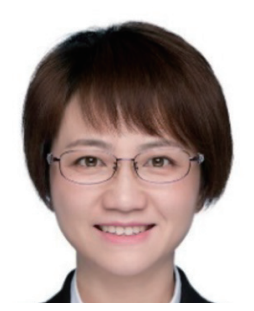

Ying Zhou received her B.S. and M.S. degrees from Xi'an University of Architecture and Technology, China, in 1999 and 2002, respectively. She received her Ph.D. degree from Tongji University, China, in 2005. From 2006 to 2009, she was a lecturer at Tongji University. From 2009 to 2012, she was an associate professor at Tongji University. Since 2012, she has been a professor at Tongji University. Her research interests are in seismic and energy dissipation technologies for engineering structures.

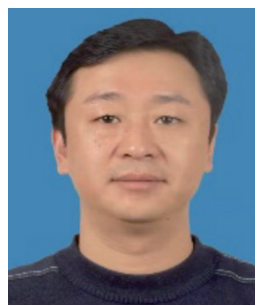

Weishi Zhao received his B.S. degree from Changchun University of Technology, China, in 2008. In 1995, he entered Changchun Oil Transportation Communication Company. Since 2018, he has been the chief engineer at the East China branch of China National Petroleum Corporation.

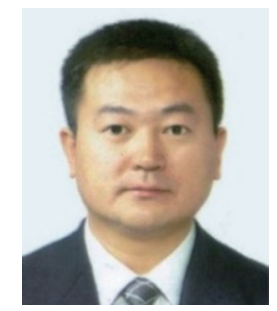

Xuefeng Li graduated from Shenyang Institute of Engineering, China, in 1999. He received his M.E. and D.E. degrees from Fukuoka Institute of Technology, Fukuoka, Japan, in 2004 and 2007, respectively. From 2007 to 2013, he was a postdoctoral and assistant researcher at Waseda University, Kitakyushu, Japan. Since 2010, he has been an associate professor at Tongji University. His research interests are in sensors and NDT. 